\title{
Application of infrared thermography for early assessment of burn wound depth in children- a preliminary study
}

\author{
by M. Janicki*, W. Tylman*, W. Kuzanski** and A. Napieralski*
}

* Dept. of Microelectronics and Computer Science, Technical University of Lodz, Wolczanska 221/223, 90-924 Lodz, Poland, janicki@dmcs.pl

** Department of Paediatric Surgery and Oncology, Medical University of Lodz, Sporna 36/50, 91-738 Lodz, Poland

\begin{abstract}
This paper discusses the problem of children's burn wound depth assessment by the analysis of infrared images. This problem is very important from the clinical point of view since the proper and early assessment of the burn wound depth determines the choice of a treatment method. Here, infrared images are always analysed together with visible light images of wounds providing a surgeon with detailed maps of wound areas. The main difficulty consists in the proper overlay of the images and the establishing of the correct temperature threshold for the classification of a wound. All the infrared image analyses are validated with the histopathological evaluation of biopsy material.
\end{abstract}

\section{Introduction}

Children are the population which is the most exposed to thermal injury. The problem of the early assessment of burn wound depth is extremely important because it determines the choice of a particular treatment method. Namely, superficially bunt skin can heal in a natural way whereas the deeply damaged one has to be excised and covered with the split thickness skin graft within a few days after a thermal injury [1]-[2]. The current practice is to combine the clinical assessment of a wound by a surgeon with the histopathological evaluation of biopsy material. Such an assessment procedure is invasive for a young patient and it is subject to human errors.

This paper proposes a new method of burn wound depth assessment in children which is both non-invasive and allows the elimination of the human factor. The proposed method uses for the assessment of the burn wound depth recorded infrared and visible light images of a wound. After the overlay of the images, the classification of a burn wound is carried out by the proper temperature thresholding of the infrared image. The exact temperature threshold value was established comparing with the traditional evaluation of biopsy material on the statistically significant number of patients. The following section provides the information on the methods for burn wound assessment as well as it describes the previous research on the use of infrared thermography in the investigation of burn wounds. Then, the current research results are presented in detail providing important conclusions and indications for further development of the method.

\section{Theoretical background}

The early clinical assessment of the burn wound depth still remains the key problem in the treatment of burn injuries. Certainly, the physical thickness of the necrotic tissue cannot serve as a useful wound classification parameter because the skin of a person has different depth at various places. Now, the commonly accepted classification of burn wounds, originally proposed by Jackson in early seventies [3], takes into account the time of wound healing physiology and relates the depth of a wound to the necrosis of particular skin layers [4]-[5].

The early assessment of burn wound depth allowing the precise determination of necrosis limits is necessary since it influences the decision of a surgeon on which areas should be left for the conservative treatment and which ones should undergo the surgical excision and grafting. According to the Jackson classification, the limit for the decision is set between the degrees IIa and IIb. Currently, the errors in the early assessment of burn wounds amount typically to $30 \%$ and the results might differ significantly not only among individual burn wound treatment clinics but they depend also on a particular physician performing the assessment. Thus, there exists an urgent need to develop a new and possibly noninvasive assessment method which would allow the objective and robust classification of the burn wound depth.

Technically, except for the usual examination of a wound by a physician, the assessment of burn wound depth can be carried out by various methods such as Doppler imaging, fluorescence spectroscopy or ultrasonography, but still the most accurate is the histopathological evaluation of biopsy material. Unfortunately, with this method it is not possible to obtain the precise necrosis map and determine the exact area where the necrectomy should be performed. The other of the above-mentioned methods are either not accurate or simply too expensive. Thus, the only reasonable alternative for the assessment of the burn wound depth is the infrared thermography, which allows the simultaneous determination of the necrosis depth and its area. This method uses the fact that a human body removes up to $60 \%$ of its energy through the skin emitting the infrared radiation in the range of 3-20 micrometers. The skin temperature depends on the blood flow rate, the sweat evaporation and the ambient temperature. Thus, the infrared thermography, with its $70 \mathrm{mK}$ temperature resolution, seems to be well suited for the accurate quantitative measurements of skin temperature [6]. 
The possibility of the use of infrared thermography for the burn wound depth assessment was reported already in the mid-seventies [7]. The influence of the reduced vascular blood circulation in thermally damaged tissues on skin temperature was confirmed both experimentally [8]-[10] and in clinical research [11]-[12]. However, the applicability of the infrared thermography was practically demonstrated only in the nineties when the thermographic classification method was successful in $92 \%$ of the analyzed cases [13]. There, the validation of the method was carried out by the comparison with the healing time of a wound. Unfortunately, taking into account that the surgery should take place very soon after a thermal injury, such a retrospective validation of a method is not possible in an everyday clinical practice. Thus, in the research presented here the results of infrared image analyses were compared only with the histopathological evaluation of biopsies. The preliminary research results obtained by the authors were very promising and demonstrated that the change of skin temperature in a deeply burn wound due to the damage in the blood microcirculation can exceed even $3.8 \mathrm{~K}$ with respect to the healthy skin [14].

Summarizing this section it should be said that burn injuries damage the microcirculation of blood in the wound tissues, which is reflected in the change of skin temperature. Thus, the infrared thermography is a well-suited method for the assessment of burn wound depth. The analysis of skin temperature differences within the registered infrared images should render possible the classification of the burn wound depth by segmenting each image into individual areas having a certain range of temperatures. The segmentation, as showed later on in this paper, can be carried out by determining the correct temperature threshold level allowing robust distinction of the between the burns of degrees IIa and IIb.

\section{Practical results}

The authors of this paper have a relatively long experience with the application of infrared thermography for the clinical research on burn wounds. The first infrared system for the assessment of burn wounds, based on the scanning Agema AGA 760 camera, was developed by one of the co-authors in the Medical Academy of Lodz already in 1985. Then, the research was continued using the Inframetrics SC1000 camera. The latest version of the infrared system developed by the authors for the Department of Paediatric Surgery and Oncology of the Medical University of Lodz is based on the modern FLIR P660 camera featuring a built-in visible light channel, hence allowing the storage of both infrared and visible light images in JPEG files. This type of the camera was supposed to allow fully automatic fusion and overlay of the two kinds of images. Unfortunately, this was not possible using the software provided by the infrared camera manufacturer and the authors had to develop their own software solution for the system [15]-[16], which is briefly described in the following subsection. Then, the preliminary results of the medical research are presented in detail.

\subsection{Software development}

The software developed by the authors not only allows a semi-automatic matching between infrared and visible light images of a burn wound but also renders possible numerous analyses of the images which are useful for a surgeon. Generally, the software uses as an input infrared images recorded in the FLIR JPEG-like format and visible light images saved in the standard JPEG format. The main input is the infrared image and the visible light one plays only an auxiliary role providing means to map burn wound areas from the infrared image to the body areas in the visible light one as seen by human eyes. The user interface should render possible the display of both images next to each other, thus making their comparison straightforward.

Therefore, the first problem tackled by the authors during the software development was related to the imperfect overlay between infrared and visible light images. The reason for that is that the camera uses different lenses to capture the images. Moreover the lenses are not coaxially located. As a result, as shown in figure 1, the two types of images have completely different field and angle of view. Thus, for the precise overlay of the images some processing is required to match them. The processing consists of two consecutive steps: the determination of common reference points in both images and the application of suitable image transformations.

The image transformation coefficients can be computed only when a certain number of reference point pairs are identified so that the points in each pair correspond exactly to the same physical locations in both types of images. As a rule, the more pairs are identified, the more parameters can be defined in the transformation and consequently more complex image deformations can be corrected. The particular software developed by the authors uses only three pairs of points, but it is sufficient to perform an image transformation. The reference points can be selected manually or adopting a fully automatic approach based on the determination of some identical features in visible light and infrared images. The developed software uses a mixed approach because the detection of reference points is automatic but the features are predefined shapes placed in different locations on the patient's skin. These predefined shapes are marking pegs in the form of black squares having the area of a square centimetre (see the figures).

The procedure for the detection of marking pegs consists of several steps which are similar for both infrared and visible light images. The first step of the procedure is the conversion of images to a grayscale equivalent format. Next, the edge detection procedure is applied allowing the detection of objects differing significantly in brightness from their neighbourhood, such as the reference black squares. This is followed by the application of a sequence of dilatation and erosion filtering algorithms which are supposed to discern individual objects in the images and increase the chance that they are limited by a joint boundary. The dilatation filter grows objects in the images, consequently filling the gaps which might appear in the boundaries of the reference squares. On the other hand, the erosion filter removes small isolated objects, thus reducing noise and smoothing out the boundaries of the squares. 

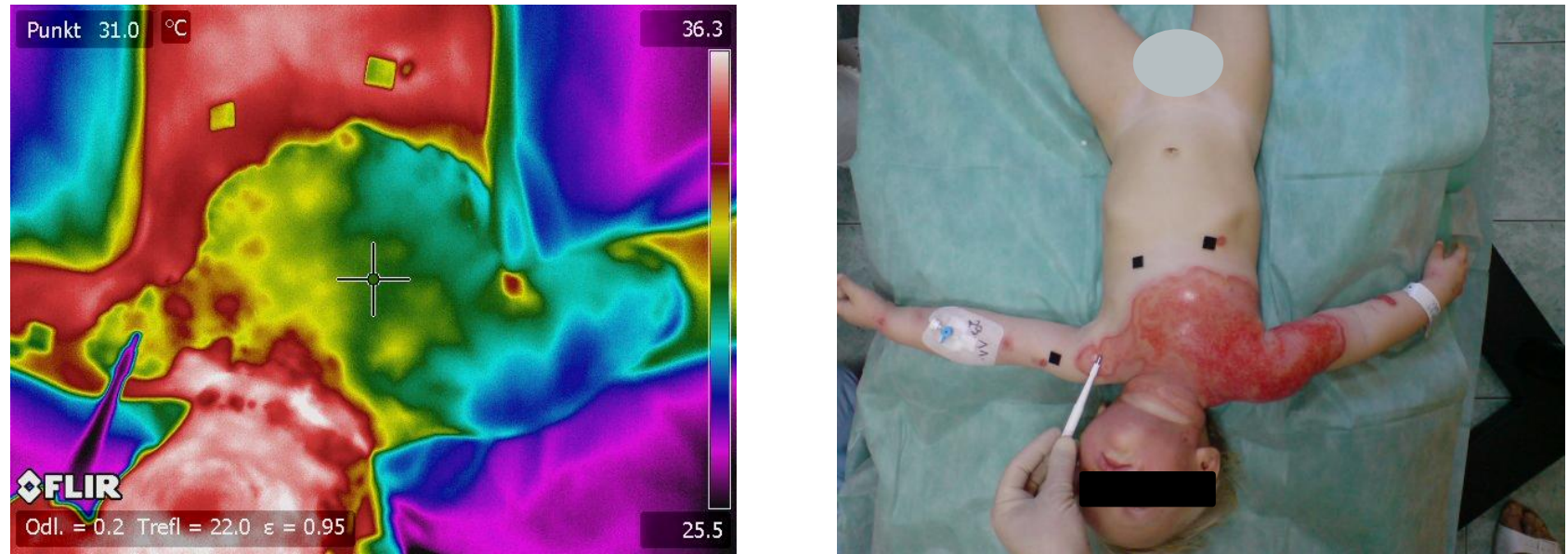

Fig. 1. Original recorded images.

Then, the resulting pre-processed images are passed to the blob detector which labels regions corresponding to individual objects larger than a predefined threshold area. This algorithm produces a list of large objects including the three black squares. The next step is the exclusion from the list of the objects which significantly differ from the square shape. For this purpose, every object in the list is tested for being quadrilateral and having sides and angles of similar values. The objects which pass the test are sorted by their area. The final step of the algorithm is the search through the list of remaining objects for three shapes having similar areas. When such a set is found, the entire detection procedure is finished.

Before the actual image transform can be performed, the reference points in infrared images have to be related to their counterparts in visible light images. For three reference points in a figure, there exist six possible ways of pairing the identified points. Thus, in order to select the correct solution, the Euclidean distances between the points of interest are calculated in both types of images. Then, for each pairing, the sum of distances between the points is computed and the solution producing the lowest sum of distances is selected as the correct one. This approach is justified when the mutual rotation between images is not significant, which is true in the considered case.

Finally, the three pairs of points matched in the above-described procedure allow the execution of an affine transform, i.e., a transform that can map a parallelogram onto square [17]. Numerous tests carried out on real images registered for burn wounds located in different parts of a human showed that this kind of transform is sufficient for the considered application. As far as the choice of a particular image to be transformed is concerned, it should be underlined that it is the visible light image which should undergo the transformation because, for medical diagnosis reasons, the infrared one should not be subject to any unnecessary processing. The result of the fully automatic correction procedure, presenting the transformed image from figure 1, is shown in figure 2.

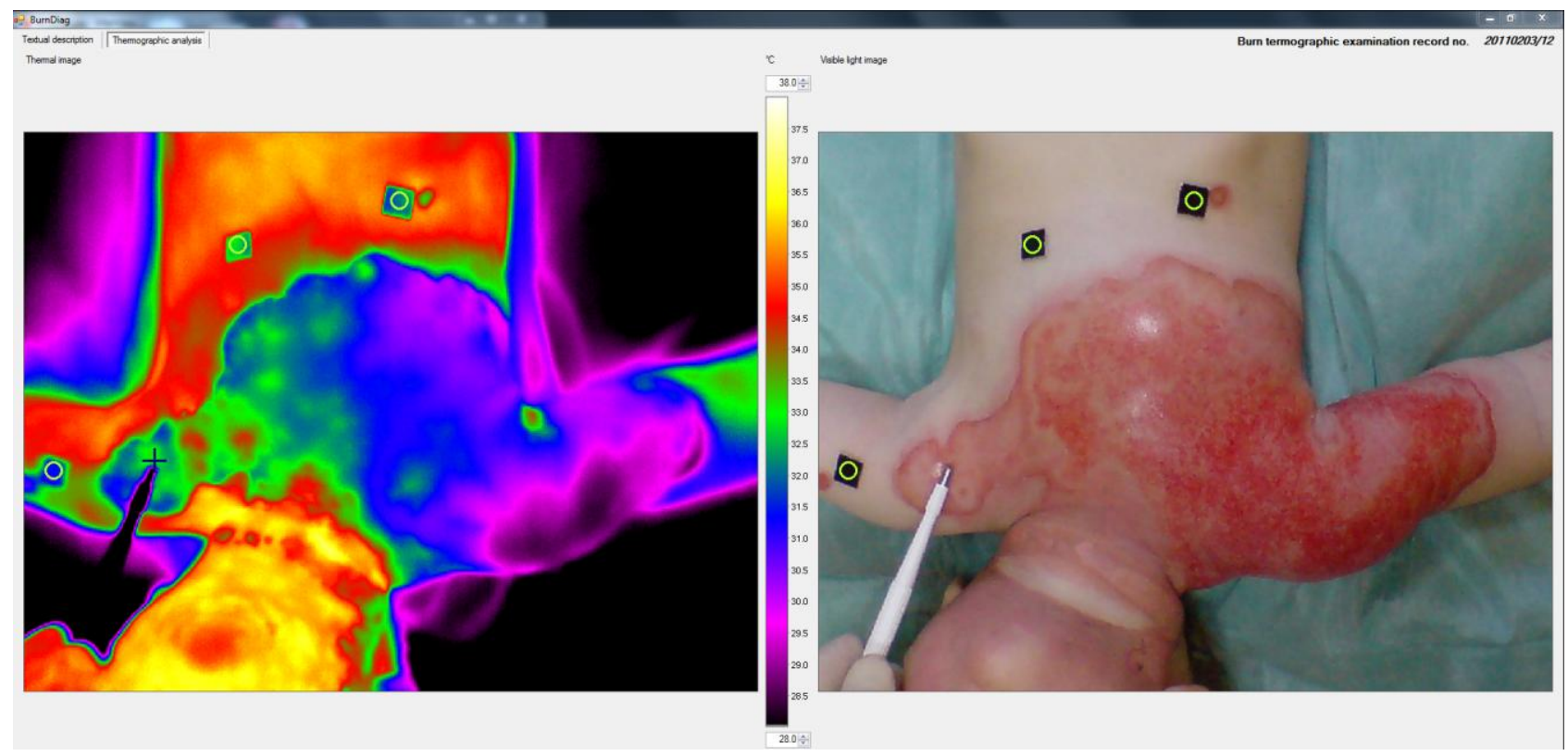

Fig. 2. Processed and correlated images. 


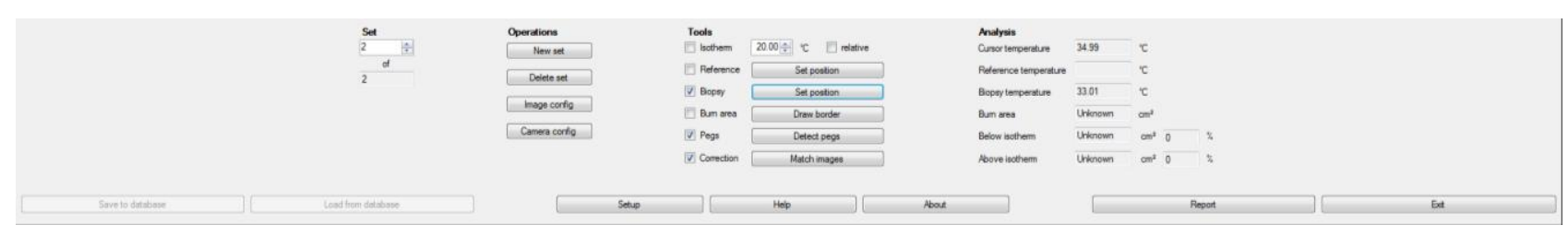

Fig. 3. Image analysis controls.

Due to the extremely low spatial resolution of infrared images (only $640 \times 480$ pixels), compared to the visible light ones, the reference squares sometimes may be too small for their reliable detection in an image. Then, when the algorithm fails to detect the reference squares or when simply marking pegs are not used during the examination, the developed software allows also manual marking of reference points in recorded images, indicating them by a simple mouse click.

Once transformed and matched, both visible light and infrared images of burn wounds can be processed further so as to extract information useful for physicians in the diagnosis process. Taking into account that all examinations have to be carried out in a hospital, the user interface was designed as simple as possible. Therefore, apart from the images, the main user interface window contains only a few additional control buttons, shown in figure 3 , activating various tools or edit boxes required for carrying out different analyses and displaying their results. These additional program features were developed in the close cooperation with the physicians directly examining children with thermal injuries. According to their detailed specifications the software assisting the diagnosis should render possible the following:

- present infrared images in a user-selected false colour palette,

- adjust their temperature range and span,

- allow freehand drawing of a closed line delimiting the wound area,

- mark biopsy locations and read their temperature,

display an isotherm and compute the surface of wound areas having temperature below and above the isotherm.

The last of the features enumerated above is very important from the point of view of a surgeon because the temperature value for which the isotherm is drawn can be defined relatively to a user-selected point in an image, what complies with the procedure of burn wound evaluation, where the burn wound treatment method is chosen based on its temperature with respect to the healthy skin.

Another additional feature of the program is that the temperature of a point indicated by the cursor in an infrared image is continuously displayed on the screen and a twin cursor is displayed in the other image pointing to the same physical location. Additionally, the current temperature range and color palette are displayed in an image, thus facilitating the image analysis using the pseudo-colour approach.

Moreover, the reporting facility was added to the developed software. It renders possible printing of examination results or including them into other documents. The reports are automatically generated at a click of the control button and they contain infrared and visible light images, the text description of a wound and patient data retrieved from the database. The reports can be generated as MS Word, MS Excel or Adobe PDF files. The data storage in the developed software complies with the regulations concerning medical images, including the DICOM standard.

Concluding, the developed software supports the diagnostic process of burn wounds, thus providing paediatric surgeons a new useful tool for early assessment of the burn depth. The ultimate result of the recorded image analyses is the detailed map of necrosis in a wound. This map, generated by the automatic creation of the isotherm dividing the burn wound area into two parts, informs a surgeon which regions need necrectomy and which ones should not be treated surgically and left for spontaneous healing. Finally, the software estimates automatically wound areas, what is beneficial for the grafting.

\subsection{Medical research}

The first problem during the examination was to establish the standard procedures to register infrared images of a burned wound in children. The examination with an infrared camera provides useful information on the burn depth only within the first few days after a thermal injury because later on the inflammatory processes occurring in the wound tissue change the original temperature distribution in a wound resulting from the blood vessels damage. Therefore, the authors decided to repeat the investigation before the surgery only 24 and 72 hours after a thermal injury.

Each time the infrared assessment of a burn wound was validated with a number of standard biopsies, which were taken only in the locations indicated by a surgeon and marked in infrared images by sterile pads of a diameter comparable with the one of the needle used for the biopsy. These pads served also as useful additional reference points during the image analyses allowing more precise alignment of visible light and infrared images. The infrared assessment of the burn wound depth showed extremely high correlation with the histopathological evaluation of the biopsy material and the clinical assessment of a wound by a physician. The results demonstrate that the temperature of superficially burned wound is only slightly changed with respect to the undamaged skin surrounding the wound. On the contrary, the deeply burned skin has the temperature lower by more than $3 \mathrm{~K}$. As far as the optimal temperature thresholding value used for the separation between the burns of degrees Ila and IIb is concerned, the experimental results show that this value should be located approximately $1.45 \mathrm{~K}$ below the healthy skin temperature. This important result has been proven on a statistically sound number of clinical cases. 
When setting the precise temperature thresholding value, one should keep in mind that if this value is set too low, some of the necrotic tissues will not be removed during a surgery and the grafting will have to be repeated. Hence, it is much safer to set this value at a bit higher level than required. Then, patients will not have to undergo an additional surgery.

\section{Conclusions}

This paper presented a new possible clinical method for the classification of the burn wound depth based on the analysis of infrared images. This method seems to be promising and compared to the traditional biopsy it is contactless and non-invasive, thus it is much more tolerable for young patients and brings the very much same results from the diagnostic point of view. Additionally, the proposed method eliminates also the human factor from the diagnostic process and the results do not depend on a particular person performing the diagnostics or his current condition.

The presented research combined the quantitative thermographic examination of burn wounds in children with the original quantitative histometrical assessment of biopsy punches taken at the same time from the examined wounds, hence proving to our best knowledge for the first time the high correlation between these two investigation methods; noninvasive thermography and invasive histopathology.

Consequently, it has been made possible to draw the exact isotherm dividing a burn wound area into two parts: superficially and deeply damaged, hence offering to surgeons a new non-invasive instrument to create maps of burn wounds which allows them to make the correct, early and exact decision: to operate or not to operate on the patient. Moreover, such thermographic maps of burn wounds resulting from examinations are exact plans for surgical operations indicating places within the wound where necrotic tissues must be excised and places where surgical intervention is not required. It is also worth mentioning that the developed software for the analysis of burn wounds in children allows the complete and objective documentation of thermal injuries; including the storage of infrared and visible light images, the course of medical treatment and, the most important, it supports making the therapeutic decision which is the best for the patient.

Concluding, the main benefits of the present clinical research are as follows:

- it allowed the determination of the correlation between the change of skin temperature and the degree of burn depth (especially IIa and IIb) by comparing the results of biopsy evaluation with the infrared images in a statistically sound number of cases hence allowing significant reduction, if not complete elimination, of the number of biopsies required and consequently relieving the patient and speeding up the process of burn depth evaluation,

- it facilitated fully automatic correlation and fusion of infrared and visible light images which are taken by the same camera but with different fields of view and depth of focus. The developed software allows not only off-line analyses and reporting of recorded images, but it is a real time tool used to process and analyze the images which produces the wound maps used during grafting and calculating the surface of the wound.

Further research in this area will be focused on the precise determination of the temperature value which should be used for the thresholding of the infrared images. Moreover, taking into account that the software used for the image processing is intended to be used mainly by the medical personnel, the image segmentation process should be fully automated and the user interface should be kept as simple as possible. Tests in hospital environment will determine the need for subsequent improvements of the software.

\section{ACKNOWLEDGEMENTS}

The research presented in this paper was supported by the grant of the Polish Ministry of Scientific Research and Higher Education No. N515 2423 37. The clinical study was performed with the approval of the Commission of Bioethics of the Medical University of Lodz in Poland No. RNN/16/11/KE issued on January $18^{\text {th }}, 2011$.

\section{REFERENCES}

[1] Still J.M.,. Law E.J, Belcher K., Thiruvaiyarv D., "Decreased Length of Hospital Stay by Early Excision and Grafting of Burns". Southern Medical Journal, vol. 89, pp. 578-582, 1996.

[2] Kamolz L.-P., Kitzinger H.B., Andel H., Frey M., "The Surgical Treatment of Acute Burns". European Surgery, vol. 38, pp. 417-423, 2006.

[3] Jackson D.M., "In Search of An Acceptable Burn Classification". British Journal of Plastic Surgery, vol. 23, pp. 219-226, 1970.

[4] Hendricks W.M., "The Classification of Burns". Journal of the American Academy of Dermatology, vol. 22, pp. 838-839, 1990.

[5] Heimbach D., Engrav L., Grube B., Marvin J., "Burn Depth: A Review”. World Journal of Surgery, vol. 16, pp. 10-15, 1992.

[6] Maldague P., "Theory and Practice of Infrared Technology for Non-destructive Testing". Wiley, 2001.

[7] Berger A., Dahlberg B., Gebhard W., Seidl K., "The Experimental and Clinical Use of Colour Thermography in The Evaluation of The Extent and Depth of Burns. Chirurgia Plastica, vol. 3, pp. 135-142, 1975. 
[8] Renkielska A., Nowakowski A., Kaczmarek M., Dobke M.K., Grudzinski J., Karmolinski A., Stojek W., "Static Thermography Revisited - An Adjunct Method for Determining The Depth of The Burn Injury". Burns, vol. 31, pp. 768-775, 2005.

[9] Renkielska A., Nowakowski A., Kaczmarek M., Ruminski J., "Burn Depths Evaluation Based on Active Dynamic IR Thermal Imaging - A Preliminary Study”. Burns, vol. 32, pp. 867-875, 2006.

[10] Ruminski J., Kaczmarek M., Renkielska A., Nowakowski A., "Thermal Parametric Imaging in The Evaluation of Skin Burn Depth". IEEE Transactions on Biomedical Engineering, vol. 54, pp. 303-312, 2007.

[11] Hackett M.E., "The Use of Thermography in The Assessment of Depth of Burn and Blood Supply of Flaps, with Preliminary Reports on Its Use in Dupuytren's Contracture and Treatment of Varicose Ulcers". British Journal of Plastic Surgery, vol. 27, pp. 311-317, 1974.

[12] Mason B.R., Graff A.J., Pegg S.P., "Colour Thermography in The Diagnosis of The Depth of Burn Injury". Burns Including Thermal Injury, vol. 7, pp. 197-202, 1981.

[13] Cole R.P., Jones S.G., Shakespeare P., "Thermographic Assessment of Hand Burns". Burns, vol. 16, pp. 60-63, 1990.

[14] Janicki M., Kuzanski W., Kulesza Z., Napieralski A., "Application of Infrared Thermography for The Assessment of Burn Wounds Depth". Thermology International, vol. 21, pp. 99-101, 2011.

[15] Tylman W., Janicki M., Napieralski A., "Computer-Aided Approach to Evaluation of Burn Wounds", Proceedings of 18th International Conference Mixed Design of Integrated Circuits and Systems MIXDES, Gliwice (Poland), pp. 653-656, 2011.

[16] Tylman W., Janicki M., Napieralski A., "Automatisation of Computer-Aided Burn Wounds Evaluation", Accepted for 19th International Conference Mixed Design of Integrated Circuits and Systems MIXDES, Warsaw (Poland), 2011.

[17] Zitova B., Flusser J., "Image Registration Methods: A Survey". Image and Vision Computing, vol. 21, pp. 9771000, 2003. 